\title{
FOLIAR APPLICATION OF THIAMIN STIMULATES THE GROWTH, YIELD AND BIOCHEMICAL COMPOUNDS PRODUCTION OF CORIANDER AND FENUGREEK
}

\author{
Mohmmad Hossein AMINIFARD ${ }^{1 *}$, Abbas JORKESH $^{2}$, Hamid-Reza FALLAHI $^{1}$, \\ Khosro ALIPOOR ${ }^{2}$ \\ ${ }^{1}$ Faculty of Agriculture, University of Birjand, \\ 9718837975 Birjand, Iran \\ ${ }^{2}$ Faculty of Agriculture, University of Guilan, Rasht, Iran \\ Received: January 2018; Accepted: April 2018
}

\begin{abstract}
This study was aimed to investigate the effect of thiamine (Th) foliar application on some morphophysiological aspects in coriander and fenugreek. Three levels of Th (250, 500 and $750 \mathrm{ppm})$ plus control treatment (distilled water) were studied under controlled greenhouse conditions. All the levels of Th, in particular the concentration of $500 \mathrm{ppm}$, increased the vegetative growth of coriander. 1000-grain weight, contents of nitrogen and phosphorus, carotenoid and chlorophyll b contents were the highest at a concentration of $750 \mathrm{ppm}$. Fenugreek vegetative growth also responded positively to the application of Th especially at a concentration of 750 but also $500 \mathrm{ppm}$ increased the content of leaf nitrogen, chlorophyll b, carotenoids, phenolics and antioxidant activity. Overall, the performance of coriander and fenugreek was improved by the application of Th foliar at all levels.
\end{abstract}

Key words: chlorophyll, grain weight, nitrogen content, phenolic compounds, total antioxidants

\section{INTRODUCTION}

Vitamins as bio-regulators can at certain concentrations exert a profound effect upon plant grow through regulation of primary and secondary metabolism (Abd El-Aziz et al. 2007; Amin \& Ismail 2015). Thiamine (Th), as a water-soluble vitamin, is produced by plants and microbes. This compound occurs either as free and in phosphorylated forms Th-monophosphate, Th-pyrophosphate and Th-triphosphate, (Bahuguna et al. 2012). Th-pyrophosphate acts as a cofactor for enzymes involved in metabolic processes such as acetyl-CoA production, tricarboxylic acid cycle, glycolysis, pentose phosphate pathway, citric acid and Calvin cycles (Raschke et al. 2007; Tunc-Ozdemir et al. 2009; Boubakri et al. 2012). Th is also involved in tolerance to DNA damage (Raschke et al. 2007). In addition, the exogenous application of Th increases the plant resistance against biotic and abiotic stresses such as salinity, extreme temperatures and plant pathogens (Bahuguna et al. 2012). The positive impact of Th has been previously proved on growth, yield, photosynthesis rate and nutrients, pigments, oil and protein contents in many plants such as $\mathrm{Lu}$ pinus termis (El-Awadi et al. 2016), Vicia faba (Hamada \& Khulaef 2000), Gladiolus grandiflorus (Sajjad et al. 2015; Hashish et al. 2015), Matricaria recutita (Ranjbar et al. 2014), Thymus vulgaris (Reda et al. 2005), Calendula officinalis (Soltani et al. 2014) and Ocimum basilicum (Fallahi et al. 2018). However, there is no scientific report about the foliar application of Th in the two multipurpose species, fenugreek and coriander.

Coriander (Coriandrum sativum L.) is an annual herb from the Apiaceae family, rich in minerals and vitamins, which has many food applications (Hassan \& Mahfouz 2012). Its green leaves possess a unique aroma and are also used as vegetable and food flavoring. The seeds of coriander are used as 
food, drugs, cosmetics and perfumery. Also, the seeds and leaves have many medicinal applications. In addition, coriander seed oil is rich in petroselinic acid, an important and unusual fatty acid (Neffati \& Marzouk 2008). Fenugreek (Trigonella foenumgraecum L.) also is an annual medicinal and spicy herb belonging to the Fabaceae family.Its fresh green leaves are consumed as vegetable. Leaves and seeds of fenugreek are used for flavoring and as a spice in food preparation. Moreover, this herb has a wide range of medicinal uses as a treatment in inflammation, tumors, metabolic disorders and cardiovascular diseases (Kakani \& Anwer 2012; Mandal \& DebMandal 2016).

Given the stimulatory role of vitamins in plant performance and the lack of scientific research on the effect of these bio-regulators on coriander and fenugreek, this study aimed to investigate the effects of Th foliar application on some growth, yield, physiological and biochemical traits of the two mentioned plants.

\section{MATERIALS AND METHODS}

\section{Experimental design and treatments}

In this research, the effect of foliar application of 0 , 250, 500 and $750 \mathrm{ppm}$ of thiamin (Th) on growth, yield, chlorophyll content and some biochemical indices of coriander and fenugreek was investigated. For this purpose, two separate pot experiments were carried out at research greenhouse of University of Guilan, Iran. The experiments were conducted based on a randomized complete design with three replications (three pots for each Th level). Application by spraying was started from the four-leaf stage (around 6 February) and done once every two weeks in the morning, up to early May (for about 3 months with 6 dates of spraying). In each spraying time, 20 $25 \mathrm{ml}$ of the related solution was used per pot plant to completely wet the leaves.

\section{Agronomic practices}

Seed sowing (Isfahan landrace), obtained from Isfahan-PakanBazr company, was done on 6 January in the pots with $33 \mathrm{~cm}$ diameter and $22 \mathrm{~cm}$ height. The seed bed was a mixture of soil, cow manure and sand with the ratio of $2: 1: 1$. The main soil characteristics are shown in Table 1. No additional fertilization was used during the plants' growth. Irrigation was done in intervals of 10 days and plant thinning was performed 20 days after emergence, so that 6 plants were kept in each pot. During the plant growth cycle, the greenhouse temperature was adjusted to 25 and $15^{\circ} \mathrm{C}$ during day and night, respectively. Photoperiod of 16 hours light and 8 hours of darkness was used in the experimental greenhouse. $\mathrm{CO}_{2}$ concentration and relative humidity were $350 \mathrm{ppm}$ and $40 \%$, respectively.

Table 1. Chemical properties of soil used in pot experiment for coriander and fenugreek

\begin{tabular}{ccccccc}
\hline $\begin{array}{c}\text { Organic } \\
\text { carbon } \\
(\%)\end{array}$ & $\begin{array}{c}\text { Nitro- } \\
\text { gen }(\%)\end{array}$ & $\begin{array}{c}\text { Cal- } \\
\text { cium } \\
(\%)\end{array}$ & $\begin{array}{c}\text { Phos- } \\
\text { phorous } \\
(\%)\end{array}$ & $\begin{array}{c}\text { Potas- } \\
\text { sium } \\
(\%)\end{array}$ & pH & Texture \\
\hline 1.2 & 1 & 0.48 & 0.19 & 0.55 & 7.1 & $\begin{array}{c}\text { loam } \\
\text { sandy }\end{array}$ \\
\hline
\end{tabular}

\section{Measurement of vegetative and reproductive traits}

On May 4, three plants were cut in each pot for the determination of some vegetative indices of coriander. The measured traits were plant height, leaf fresh and dry yield, root fresh and dry weight as well as stem diameter and fresh and dry weight of stems. The remaining three plants were used to determine some reproductive indices including the number of umbel per plant, number of umbellets per umbel and thousand seed weight on 28 May. The same sample sizes were used for fenugreek for the evaluation of vegetative and reproductive traits, such as plant height, leaf weight, root weight, stem weight, number of lateral branches, pod length, pod weight, number of pod per plant, number of seed per plant and 1000-grain weight.

\section{Chemical analysis}

In both plants under study, the pigments content (carotenoid, chlorophyll a and b) was determined by the method described by Mínguez-Mosquera and Pérez-Gálvez (1998). To measure the percentage of leaf nitrogen, phosphorous and calcium, the method of Jones (2001) was used. The method of Bakhshi and Arakawa (2006) was applied for the extraction to determine the phenolic compounds and antioxidant capacity in leaves. Phenols were analyzed by using the method of Folin-Ciocalteau reported by Tavarini et al. (2008). For this purpose, the absorbance at $760 \mathrm{~nm}$ was measured using 
a spectrophotometer (T80 + PG Instrument UV/Vis Spectrometer) and the values were expressed as $\mathrm{mg}$ gallic acid/100 $\mathrm{g}$ fresh weight. In addition, the antioxidant capacity was determined using DPPH free radical scavenging method as has been described by Sánchez-Moreno et al. (1999). All the chemical indices were determined at the end of the vegetative growth stage. Their determination was done on the same plants that were used for the measurement of vegetative traits, and accordingly, for each treatment (Th levels) and each replication, three plants were used for chemical analysis.

\section{Data analysis}

Data from the two greenhouse experiments were statistically analyzed using the analysis of variance (ANOVA) procedure on SAS 9.2. Means were separated via Tukey's honest significant difference test (HSD) at 5\% level of probability.

\section{RESULTS}

\section{Exogenous application of thiamin in coriander}

Thiamine foliar application significantly affected the coriander height, leaf and stem weights (Table 2). Th at a concentration of $500 \mathrm{ppm}$, resulted in the highest plants (by 29.5\%) and the highest leaf fresh and dry weight (by 24.9 and $32.8 \%$ respectively) in comparison with the control. In addition, foliar spraying of Th had a stimulating effect on stem fresh weight and diameter by 17.7 and $23.8 \%$, respectively (Table 3 ). Thousand grain weight in the plants treated with $750 \mathrm{ppm}$ of Th was higher by $29 \%$ (Table 4). Effect of Th application was significant on leaf nitrogen and phosphorous content in coriander (Table 2). At $750 \mathrm{ppm}$ Th, there were $33.8 \%$ more $\mathrm{N}$ and $22.8 \%$ more $\mathrm{P}$ in the leaves in comparison with the control (Table 4).

Table 2. Mean squares for the effect of thiamin on vegetative and reproductive indices, and some biochemical parameters in coriander leaves

\begin{tabular}{|c|c|c|c|c|c|c|c|c|}
\hline $\begin{array}{c}\text { Source } \\
\text { of variation } \\
\end{array}$ & df & Plant height & $\begin{array}{l}\text { Leaf fresh } \\
\text { weight }\end{array}$ & $\begin{array}{l}\text { Leaf dry } \\
\text { weight }\end{array}$ & $\begin{array}{l}\text { Root fresh } \\
\text { weight }\end{array}$ & $\begin{array}{l}\text { Root dry } \\
\text { weight }\end{array}$ & $\begin{array}{c}\text { Stem fresh } \\
\text { weight }\end{array}$ & $\begin{array}{c}\text { Stem dry } \\
\text { weight }\end{array}$ \\
\hline Treatment & 3 & $85.55^{*}$ & $1.22^{*}$ & $0.09^{*}$ & $0.02^{\text {ns }}$ & $0.0020^{\mathrm{ns}}$ & $0.41^{*}$ & $0.030^{*}$ \\
\hline Error & 8 & 15.75 & 0.25 & 0.01 & 0.01 & 0.0006 & 0.09 & 0.007 \\
\hline SD & & 3.84 & 0.48 & 0.12 & 0.09 & 0.02 & 0.29 & 0.08 \\
\hline $\mathrm{CV}$ & 11 & 8.21 & 7.81 & 9.49 & 8.66 & 10.06 & 5.63 & 5.82 \\
\hline $\begin{array}{c}\text { Source } \\
\text { of variation }\end{array}$ & df & $\begin{array}{l}\text { Stem diame- } \\
\text { ter }\end{array}$ & $\begin{array}{l}\text { Number } \\
\text { of umbels } \\
\text { per plant }\end{array}$ & $\begin{array}{c}\text { Number } \\
\text { of umbellets } \\
\text { per umbel }\end{array}$ & $\begin{array}{c}1000 \text { grains } \\
\text { weight }\end{array}$ & Nitrogen $\%$ & \multicolumn{2}{|c|}{ Phosphorus \% } \\
\hline Treatment & 3 & $0.190^{* *}$ & $0.55^{\mathrm{ns}}$ & $0.66^{\mathrm{ns}}$ & $3.97^{* *}$ & $0.28^{*}$ & \multicolumn{2}{|c|}{$0.008^{*}$} \\
\hline Error & 8 & 0.008 & 0.50 & 0.50 & 0.18 & 0.04 & \multicolumn{2}{|c|}{0.002} \\
\hline SD & & 0.08 & 0.68 & 0.68 & 0.42 & 0.18 & \multicolumn{2}{|c|}{0.04} \\
\hline $\mathrm{CV}$ & 11 & 3.22 & 14.62 & 14.14 & 4.21 & 8.53 & \multicolumn{2}{|c|}{7.19} \\
\hline $\begin{array}{c}\text { Source } \\
\text { of variation }\end{array}$ & $\mathrm{df}$ & Calcium \% & $\begin{array}{l}\text { Chlorophyll } \\
\text { a content }\end{array}$ & $\begin{array}{l}\text { Chlorophyll b } \\
\text { content }\end{array}$ & $\begin{array}{c}\text { Carotenoids } \\
\text { content }\end{array}$ & $\begin{array}{c}\text { Total phe- } \\
\text { nolics con- } \\
\text { tent }\end{array}$ & \multicolumn{2}{|c|}{$\begin{array}{c}\text { Total anti-oxidants ac- } \\
\text { tivity }\end{array}$} \\
\hline Treatment & 3 & $0.002^{\text {ns }}$ & $0.15^{\text {** }}$ & $0.050^{* *}$ & $0.38^{*}$ & $16.66^{\mathrm{ns}}$ & \multicolumn{2}{|c|}{$28.40^{\mathrm{ns}}$} \\
\hline Error & 8 & 0.010 & 0.01 & 0.005 & 0.08 & 21.08 & \multicolumn{2}{|c|}{15.54} \\
\hline SD & & 0.8 & 0.09 & 0.07 & 0.26 & 4.45 & \multicolumn{2}{|c|}{3.69} \\
\hline $\mathrm{CV}$ & 11 & 12.78 & 8.20 & 11.87 & 9.90 & 6.88 & \multicolumn{2}{|c|}{7.08} \\
\hline
\end{tabular}

*,**Significant at $5 \%$, and $1 \%$ probability level, respectively 
Table 3. The effect of thiamin foliar application on some vegetative growth indices (in brackets percent of increase compared with control)

\begin{tabular}{|c|c|c|c|c|c|c|c|c|}
\hline $\begin{array}{l}\text { Thiamin } \\
\text { concentra- } \\
\text { tion (ppm) }\end{array}$ & $\begin{array}{l}\text { Plant height } \\
(\mathrm{cm})\end{array}$ & $\begin{array}{l}\text { Leaf fresh } \\
\text { weight } \\
\left.\text { (g.plant }{ }^{-1}\right) \\
\end{array}$ & $\begin{array}{c}\text { Leaf dry } \\
\text { weight } \\
\left.\text { (g.plant }{ }^{-1}\right) \\
\end{array}$ & $\begin{array}{l}\text { Root fresh } \\
\text { weight } \\
\left(\text { g.plant }{ }^{-1}\right) \\
\end{array}$ & $\begin{array}{l}\text { Root dry } \\
\text { weight } \\
\left.\text { (g.plant }{ }^{-1}\right) \\
\end{array}$ & $\begin{array}{l}\text { Stem fresh } \\
\text { weight } \\
\left(\text { g.plant }{ }^{-1}\right) \\
\end{array}$ & $\begin{array}{l}\text { Stem dry } \\
\text { weight } \\
\left.\text { (g.plant }^{-1}\right) \\
\end{array}$ & $\begin{array}{c}\text { Stem diame- } \\
\text { ter } \\
(\mathrm{mm}) \\
\end{array}$ \\
\hline 0 & $42.7 \pm 4.0 \mathrm{~b}$ & $5.70 \pm 0.52 \mathrm{~b}$ & $1.25 \pm 0.19 \mathrm{~b}$ & $1.20 \pm 0.090 \mathrm{a}$ & $0.21 \pm 0.032 \mathrm{a}$ & $4.95 \pm 0.33 \mathrm{~b}$ & $1.42 \pm 0.088 \mathrm{~b}$ & $2.52 \pm 0.04 \mathrm{c}$ \\
\hline 250 & $\begin{array}{c}46.3 \pm 3.5 \mathrm{ab} \\
(8.4) \\
\end{array}$ & $\begin{array}{c}6.10 \pm 0.27 \mathrm{ab} \\
(7.01)\end{array}$ & $\begin{array}{c}1.36 \pm 0.06 \mathrm{ab} \\
(8.8)\end{array}$ & $\begin{array}{c}1.18 \pm 0.055 \mathrm{a} \\
(-1.6)\end{array}$ & $\begin{array}{c}0.23 \pm 0.030 \mathrm{a} \\
(9.5)\end{array}$ & $\begin{array}{c}5.22 \pm 0.27 \mathrm{ab} \\
(5.4)\end{array}$ & $\begin{array}{c}1.66 \pm 0.101 \mathrm{a} \\
(14.4)\end{array}$ & $\begin{array}{c}2.77 \pm 0.12 b c \\
(9.9)\end{array}$ \\
\hline 500 & $\begin{array}{c}55.3 \pm 2.5 \mathrm{a} \\
(29.5) \\
\end{array}$ & $\begin{array}{c}7.12 \pm 0.51 \mathrm{a} \\
(24.9) \\
\end{array}$ & $\begin{array}{c}1.66 \pm 0.13 \mathrm{a} \\
(32.8) \\
\end{array}$ & $\begin{array}{c}1.25 \pm 0.060 \mathrm{a} \\
(4.1)\end{array}$ & $\begin{array}{c}0.27 \pm 0.015 \mathrm{a} \\
(28.5)\end{array}$ & $\begin{array}{c}5.83 \pm 0.22 \mathrm{a} \\
(17.7)\end{array}$ & $\begin{array}{c}1.48 \pm 0.045 \mathrm{ab} \\
(4.2)\end{array}$ & $\begin{array}{c}3.12 \pm 0.10 \mathrm{a} \\
(23.8)\end{array}$ \\
\hline 750 & $\begin{array}{c}49.0 \pm 2.3 \mathrm{ab} \\
(14.7)\end{array}$ & $\begin{array}{c}6.76 \pm 0.62 \mathrm{ab} \\
(18.5)\end{array}$ & $\begin{array}{c}1.50 \pm 0.12 \mathrm{ab} \\
(20)\end{array}$ & $\begin{array}{c}1.39 \pm 0.178 \mathrm{a} \\
(15.8)\end{array}$ & $\begin{array}{c}0.27 \pm 0.017 \mathrm{a} \\
(28.5)\end{array}$ & $\begin{array}{c}5.46 \pm 0.35 \mathrm{ab} \\
(10.3)\end{array}$ & $\begin{array}{c}1.48 \pm 0.104 \mathrm{ab} \\
(4.2)\end{array}$ & $\begin{array}{c}2.94 \pm 0.08 \mathrm{ab} \\
(16.6)\end{array}$ \\
\hline
\end{tabular}

Means with the same letter(s) within a column are not significantly different $(\mathrm{P} \leq 0.05)$ based on Tukey's test

Table 4. The effect of thiamin foliar application on some reproductive indices and contents of $\mathrm{N}, \mathrm{P}$ and $\mathrm{Ca}$ in coriander leaves (in brackets percent of increase compared with control)

\begin{tabular}{|c|c|c|c|c|c|c|}
\hline $\begin{array}{c}\text { Thiamin } \\
\text { concentration } \\
(\mathrm{ppm})\end{array}$ & $\begin{array}{c}\text { Number } \\
\text { of umbels } \\
\text { per plant }\end{array}$ & $\begin{array}{c}\text { Number } \\
\text { of umbellets } \\
\text { per umbel }\end{array}$ & $\begin{array}{l}\text { 1000-grains } \\
\text { weight (g) }\end{array}$ & $\begin{array}{l}\text { Leaf nitrogen } \\
(\%)\end{array}$ & $\begin{array}{l}\text { Leaf phospho- } \\
\quad \text { rus }(\%)\end{array}$ & $\begin{array}{l}\text { Leaf calcium } \\
(\%)\end{array}$ \\
\hline 0 & $4.7 \pm 0.57 \mathrm{a}$ & $4.7 \pm 0.57 \mathrm{a}$ & $08.96 \pm 0.37 \mathrm{~b}$ & $2.16 \pm 0.025 \mathrm{~b}$ & $0.57 \pm 0.063 \mathrm{~b}$ & $0.92 \pm 0.035 \mathrm{a}$ \\
\hline 250 & $\begin{array}{c}4.3 \pm 0.57 \mathrm{a} \\
(-8.5) \\
\end{array}$ & $\begin{array}{c}5.7 \pm 0.58 \mathrm{a} \\
(21.3) \\
\end{array}$ & $\begin{array}{c}09.46 \pm 0.49 \mathrm{~b} \\
(5.6)\end{array}$ & $\begin{array}{c}2.57 \pm 0.369 \mathrm{ab} \\
(19.0)\end{array}$ & $\begin{array}{c}0.63 \pm 0.032 \mathrm{ab} \\
(10.5)\end{array}$ & $\begin{array}{c}0.87 \pm 0.032 \mathrm{a} \\
(-5.4)\end{array}$ \\
\hline 500 & $\begin{array}{c}5.3 \pm 0.57 \mathrm{a} \\
(12.7)\end{array}$ & $\begin{array}{c}4.7 \pm 0.56 \mathrm{a} \\
(0.0)\end{array}$ & $\begin{array}{c}10.46 \pm 0.38 \mathrm{ab} \\
(16.7)\end{array}$ & $\begin{array}{c}2.68 \pm 0.197 \mathrm{ab} \\
(24.07)\end{array}$ & $\begin{array}{c}0.65 \pm 0.020 \mathrm{ab} \\
(14.03)\end{array}$ & $\begin{array}{c}0.87 \pm 0.041 \mathrm{a} \\
(-5.4)\end{array}$ \\
\hline 750 & $\begin{array}{c}5.0 \pm 1.0 \mathrm{a} \\
(6.4)\end{array}$ & $\begin{array}{c}5.0 \pm 1.0 \mathrm{a} \\
(6.4)\end{array}$ & $\begin{array}{c}11.56 \pm 0.44 \mathrm{a} \\
(29.0)\end{array}$ & $\begin{array}{c}2.89 \pm 0.131 \mathrm{a} \\
(33.8)\end{array}$ & $\begin{array}{c}0.70 \pm 0.055 \mathrm{a} \\
(22.8)\end{array}$ & $\begin{array}{c}0.86 \pm 0.01 \mathrm{a} \\
(6.5)\end{array}$ \\
\hline
\end{tabular}

Note: see Table 3

Th also had a significant effect on the leaf pigments content (Table 2). Contents of pigments grew with concentration of Th up to $49 \%$ for $\mathrm{Chl}$ a at $750 \mathrm{ppm}$ and up to $67.4 \%$ at $500 \mathrm{ppm}$ for Chl b (Table 5). Th at concentration of $750 \mathrm{ppm}$ increased the leaf carotenoid content by $26 \%$. Phenolic compounds and total antioxidants activity increased after Th foliar application but differences were not significant (Table 5). Exogenous application of thiamin in fenugreek

Thiamine foliar application had a significant effect on fenugreek height, leaf dry weight, root fresh and dry weights and stem fresh weight (Table 6). All the levels of Th increased the plants height and its maximum was at 500 and 750 ppm (23.9 and 21.9\% respectively as compared with control). Leaf dry weight was enhanced significantly at $750 \mathrm{ppm}$ by $34.7 \%$ (Table 7). Likewise, root fresh and dry weight increased by 18 to $25 \%$ when sprayed with 750 or $500 \mathrm{ppm}$ Th. Stem fresh weight was significantly higher with Th 500 and 750 ppm by 22 and
$20.7 \%$. Stem dry weight and number of lateral branches were not affected (Table 7). Among the reproductive traits of fenugreek, Th imposed a significant effect on the pod length, pod weight and number of pods per plant (Table 6). Pods were longer at 500 and 750 ppm by 26 and $20.8 \%$ respectively as compared with control (Table 8). Number of pods increased with $750 \mathrm{ppm}$ Th by $118 \%$. Pods weight increased in all the Th treated plants from 48 to $96 \%$, depended on Th concentration. The weight of 1000 grains and contents of $\mathrm{P}$ and $\mathrm{Ca}$ were not affected significantly (Table 8).

Leaf nitrogen, chlorophyll b, carotenoid, phenols and antioxidants activity were significantly affected by Th foliar spraying (Table 6). Leaf nitrogen content was higher with 500 and 750 Th ppm (by 30.1 and $48.9 \%$ respectively) (Table 8). Th application of 500 and $750 \mathrm{ppm}$ also stimulated the contents of $\mathrm{Chl} b$, carotenoids and antioxidants activity from 18 to $31 \%$ (Table 9). 
Table 5. The effect of thiamin foliar application on chlorophylls, carotenoids, phenolics contents and activity of antioxidants in coriander leaves (in brackets percent of increase compared with control)

\begin{tabular}{|c|c|c|c|c|c|}
\hline $\begin{array}{l}\text { Thiamin concen- } \\
\text { tration (ppm) }\end{array}$ & $\begin{array}{l}\text { Chlorophyll a } \\
\left(\mathrm{mg} \cdot \mathrm{g}^{-1} \mathrm{f} . \mathrm{w} .\right)\end{array}$ & $\begin{array}{l}\text { Chlorophyll b } \\
\left(\mathrm{mg} \cdot \mathrm{g}^{-1} \text { f. w.) }\right.\end{array}$ & $\begin{array}{l}\text { Carotenoids } \\
\left(\mathrm{mg} \cdot \mathrm{g}^{-1} \mathrm{f} . \mathrm{w} .\right)\end{array}$ & $\begin{array}{l}\text { Total phenolics } \\
\left(\mathrm{mg} \cdot 100 \mathrm{~g} \mathrm{f} . \mathrm{w}^{-1}\right)\end{array}$ & $\begin{array}{c}\text { Total anti-oxidants } \\
\text { (\% inhibition } \\
\text { of DPPH) }\end{array}$ \\
\hline 0 & $1.06 \pm 0.055 \mathrm{~b}$ & $0.46 \pm 0.087 \mathrm{~b}$ & $2.65 \pm 0.32 \mathrm{ab}$ & $63.7 \pm 5.0 \mathrm{a}$ & $51.7 \pm 3.89 \mathrm{a}$ \\
\hline 250 & $\begin{array}{c}1.20 \pm 0.077 \mathrm{ab} \\
(13.2)\end{array}$ & $\begin{array}{c}0.60 \pm 0.075 \mathrm{ab} \\
(30.4)\end{array}$ & $\begin{array}{c}2.59 \pm 0.15 b \\
(-2.2)\end{array}$ & $\begin{array}{c}66.0 \pm 4.6 \mathrm{a} \\
(3.61)\end{array}$ & $\begin{array}{c}56.5 \pm 5.84 \mathrm{a} \\
(9.3)\end{array}$ \\
\hline 500 & $\begin{array}{c}1.41 \pm 0.110 \mathrm{ab} \\
(33.01)\end{array}$ & $\begin{array}{c}0.77 \pm 0.047 \mathrm{a} \\
(67.4)\end{array}$ & $\begin{array}{c}3.07 \pm 0.42 \mathrm{ab} \\
(15.8)\end{array}$ & $\begin{array}{c}68.0 \pm 2.6 \mathrm{a} \\
(6.75)\end{array}$ & $\begin{array}{c}55.2 \pm 2.71 \mathrm{a} \\
(6.76)\end{array}$ \\
\hline 750 & $\begin{array}{c}1.58 \pm 0.150 \mathrm{a} \\
(49.05)\end{array}$ & $\begin{array}{c}0.71 \pm 0.085 \mathrm{ab} \\
(54.3) \\
\end{array}$ & $\begin{array}{c}3.34 \pm 0.15 \mathrm{a} \\
(26.03)\end{array}$ & $\begin{array}{c}69.0 \pm 2.5 \mathrm{a} \\
(8.3)\end{array}$ & $\begin{array}{c}59.1 \pm 2.33 \mathrm{a} \\
(14.3) \\
\end{array}$ \\
\hline
\end{tabular}

Note: see Table 3

Table 6. Mean squares for the effect of different levels of thiamin on vegetative and reproductive indices, leaf nutrient and pigments content and some biochemical parameters in fenugreek

\begin{tabular}{|c|c|c|c|c|c|c|c|c|}
\hline $\begin{array}{c}\text { Source } \\
\text { of variation }\end{array}$ & $\mathrm{df}$ & Plant height & $\begin{array}{c}\text { Leaf fresh } \\
\text { weight }\end{array}$ & $\begin{array}{c}\text { Leaf dry } \\
\text { weight }\end{array}$ & $\begin{array}{l}\text { Root fresh } \\
\text { weight }\end{array}$ & $\begin{array}{c}\text { Root dry } \\
\text { weight }\end{array}$ & $\begin{array}{c}\text { Stem fresh } \\
\text { weight }\end{array}$ & $\begin{array}{c}\text { Stem dry } \\
\text { weight }\end{array}$ \\
\hline Treatment & 3 & $90.88^{*}$ & $1.31^{\mathrm{ns}}$ & $0.23^{*}$ & $0.53^{*}$ & $0.030^{*}$ & $0.85^{* *}$ & $0.01^{\mathrm{ns}}$ \\
\hline Error & 8 & 22.00 & 0.46 & 0.04 & 0.08 & 0.004 & 0.07 & 0.02 \\
\hline Std Dev & & 4.58 & 0.66 & 0.19 & 0.27 & 0.06 & 0.25 & 0.16 \\
\hline $\mathrm{CV}$ & 11 & 8.47 & 8.36 & 8.93 & 6.78 & 6.01 & 4.41 & 10.77 \\
\hline $\begin{array}{c}\text { Source } \\
\text { of variation }\end{array}$ & df & $\begin{array}{c}\text { Number of lat- } \\
\text { eral branches }\end{array}$ & $\begin{array}{c}\text { Pod mean } \\
\text { length }\end{array}$ & $\begin{array}{c}\text { Number of pod } \\
\text { per plant }\end{array}$ & $\begin{array}{c}\text { Number of seed } \\
\text { per pod }\end{array}$ & $\begin{array}{l}\text { Pod weight } \\
\text { per plant }\end{array}$ & $\begin{array}{c}\text { 1000-grain } \\
\text { weight }\end{array}$ & $\begin{array}{l}\text { Leaf nitro- } \\
\text { gen content }\end{array}$ \\
\hline Treatment & 3 & $1.19^{\mathrm{ns}}$ & $3.50^{* * *}$ & $157.19^{* *}$ & $2.52^{\mathrm{ns}}$ & $134.75^{* *}$ & $3.43^{\mathrm{ns}}$ & $0.49^{* *}$ \\
\hline Error & 8 & 0.41 & 0.26 & 2.66 & 0.91 & 3.83 & 1.19 & 0.01 \\
\hline Std Dev & & 0.53 & 0.46 & 1.60 & 0.93 & 1.81 & 1.07 & 0.08 \\
\hline $\mathrm{CV}$ & 11 & 11.56 & 4.67 & 6.97 & 6.14 & 7.96 & 6.39 & 4.75 \\
\hline $\begin{array}{c}\text { Source } \\
\text { of variation }\end{array}$ & $\mathrm{df}$ & $\begin{array}{c}\text { Phosphorous } \\
\text { content }\end{array}$ & $\begin{array}{l}\text { Calcium } \\
\text { content }\end{array}$ & Chlorophyll a & Chlorophyll b & $\begin{array}{c}\text { Carotenoids } \\
\text { content }\end{array}$ & $\begin{array}{c}\text { Total } \\
\text { phenolics }\end{array}$ & $\begin{array}{l}\text { Total anti- } \\
\text { oxidants }\end{array}$ \\
\hline Treatment & 3 & $0.003^{\mathrm{ns}}$ & $0.003^{\mathrm{ns}}$ & $0.010^{\mathrm{ns}}$ & $0.010^{* *}$ & $0.0050^{*}$ & $94.97^{* *}$ & $113.63^{*}$ \\
\hline Error & 8 & 0.002 & 0.002 & 0.006 & 0.001 & 0.0008 & 7.25 & 20.00 \\
\hline Std Dev & & 0.04 & 0.04 & 0.07 & 0.03 & 0.02 & 2.45 & 3.90 \\
\hline $\mathrm{CV}$ & 11 & 11.73 & 4.3 & 6.52 & 5.66 & 5.28 & 5.07 & 8.29 \\
\hline
\end{tabular}

$*$,**Significant at $5 \%$, and $1 \%$ probability level, respectively.

Table 7. The effect of thiamin foliar application on some vegetative growth indices in fenugreek (in brackets percent of increase compared with control)

\begin{tabular}{|c|c|c|c|c|c|c|c|c|}
\hline $\begin{array}{l}\text { Thiamin } \\
\text { concentra- } \\
\text { tion (ppm) }\end{array}$ & $\begin{array}{l}\text { Plant height } \\
(\mathrm{cm})\end{array}$ & $\begin{array}{l}\text { Leaf fresh } \\
\text { weight } \\
\left(\mathrm{g} \cdot \text { plant }^{-1}\right)\end{array}$ & $\begin{array}{l}\text { Leaf dry } \\
\text { weight } \\
\left(\mathrm{g} \cdot \text { plant }^{-1}\right)\end{array}$ & $\begin{array}{l}\text { Root fresh } \\
\text { weight } \\
\left(\mathrm{g} \cdot \text { plant }^{-1}\right)\end{array}$ & $\begin{array}{l}\text { Root dry } \\
\text { weight } \\
\left(\mathrm{g} \cdot \text { plant }^{-1}\right)\end{array}$ & $\begin{array}{l}\text { Stem fresh } \\
\text { weight } \\
\left(\mathrm{g} \cdot \text { plant }^{-1}\right)\end{array}$ & $\begin{array}{l}\text { Stem dry } \\
\text { weight } \\
\left(\mathrm{g} \cdot \text { plant }^{-1}\right)\end{array}$ & $\begin{array}{l}\text { Number } \\
\text { of lateral } \\
\text { branches } \\
\text { per plant }\end{array}$ \\
\hline 0 & $48.66 \pm 5.5 \mathrm{~b}$ & $7.37 \pm 0.57 \mathrm{a}$ & $1.90 \pm 0.10 \mathrm{~b}$ & $3.61 \pm 0.33 \mathrm{~b}$ & $1.08 \pm 0.10 \mathrm{~b}$ & $5.30 \pm 0.16 \mathrm{~b}$ & $1.50 \pm 0.16 \mathrm{a}$ & $4.7 \pm 0.57 \mathrm{a}$ \\
\hline 250 & $\begin{array}{c}53.00 \pm 5.0 \mathrm{ab} \\
(8.9)\end{array}$ & $\begin{array}{c}7.97 \pm 0.56 \mathrm{a} \\
(8.14)\end{array}$ & $\begin{array}{c}2.30 \pm 0.26 \mathrm{ab} \\
(21.05)\end{array}$ & $\begin{array}{c}4.20 \pm 0.14 \mathrm{ab} \\
(16.3)\end{array}$ & $\begin{array}{c}1.07 \pm 0.05 \mathrm{~b} \\
(-0.9)\end{array}$ & $\begin{array}{c}6.12 \pm 0.18 \mathrm{ab} \\
(15.5)\end{array}$ & $\begin{array}{c}1.57 \pm 0.21 \mathrm{a} \\
(4.66)\end{array}$ & $\begin{array}{c}6.0 \pm 0.0 \mathrm{a} \\
(27.65)\end{array}$ \\
\hline 500 & $\begin{array}{c}60.33 \pm 5.1 \mathrm{a} \\
(23.98)\end{array}$ & $\begin{array}{c}8.86 \pm 0.61 \mathrm{a} \\
(20.2)\end{array}$ & $\begin{array}{c}2.36 \pm 0.15 \mathrm{ab} \\
(24.2)\end{array}$ & $\begin{array}{c}4.53 \pm 0.29 \mathrm{a} \\
(25.48)\end{array}$ & $\begin{array}{c}1.25 \pm 0.07 \mathrm{ab} \\
(15.7)\end{array}$ & $\begin{array}{c}6.47 \pm 0.37 \mathrm{a} \\
(22.07)\end{array}$ & $\begin{array}{c}1.69 \pm 0.17 \mathrm{a} \\
(12.66)\end{array}$ & $\begin{array}{c}6.0 \pm 1.0 \mathrm{a} \\
(27.65)\end{array}$ \\
\hline 750 & $\begin{array}{c}59.33 \pm 2.5 \mathrm{a} \\
(21.92)\end{array}$ & $\begin{array}{c}8.56 \pm 0.92 \mathrm{a} \\
(16.14)\end{array}$ & $\begin{array}{c}2.56 \pm 0.25 \mathrm{a} \\
(34.7)\end{array}$ & $\begin{array}{c}4.49 \pm 0.32 \mathrm{a} \\
(24.37)\end{array}$ & $\begin{array}{c}1.27 \pm 0.04 \mathrm{a} \\
(17.6)\end{array}$ & $\begin{array}{c}6.40 \pm 0.28 \mathrm{a} \\
(20.75)\end{array}$ & $\begin{array}{c}1.63 \pm 0.13 \mathrm{a} \\
(8.66)\end{array}$ & $\begin{array}{c}5.7 \pm 0.57 \mathrm{a} \\
(21.27)\end{array}$ \\
\hline
\end{tabular}

Note: see Table 3 
Table 8. The effect of thiamin foliar application on some reproductive indices and contents of N, P and Ca in fenugreek leaves (in brackets percent of increase compared with control)

\begin{tabular}{|c|c|c|c|c|c|c|c|c|}
\hline $\begin{array}{l}\text { Thiamine } \\
\text { concentra- } \\
\text { tion (ppm) }\end{array}$ & $\begin{array}{l}\text { Pod mean } \\
\text { length } \\
(\mathrm{cm})\end{array}$ & $\begin{array}{c}\text { Number } \\
\text { of pod per } \\
\text { plant }\end{array}$ & $\begin{array}{c}\text { Number } \\
\text { of seed per } \\
\text { pod }\end{array}$ & $\begin{array}{l}\text { Pod weight } \\
\text { per plant } \\
(\mathrm{g})\end{array}$ & $\begin{array}{l}\text { weight } \\
(\mathrm{g})\end{array}$ & $\begin{array}{c}\text { Leaf } \\
\text { nitrogen } \\
(\%)\end{array}$ & $\begin{array}{c}\text { Leaf } \\
\text { phosphorus } \\
(\%)\end{array}$ & $\begin{array}{c}\text { Leaf } \\
\text { calcium } \\
(\%)\end{array}$ \\
\hline 0 & & & & $\mathrm{c}$ & - & b & & \\
\hline 250 & ( & $\mathrm{b}$ & (2 & $8 \mathrm{a}$ & $32 \mathrm{a}$ & $25 \mathrm{~b}$ & $(-5$ & $(0$ \\
\hline 50 & $\begin{array}{r}12.1 \\
(2 \\
\end{array}$ & $\begin{array}{r}28.3 \pm \\
(11 \\
\end{array}$ & $\begin{array}{r}16.7= \\
(13\end{array}$ & $\begin{array}{r}23.3= \\
(4\end{array}$ & $\begin{array}{r}17.9 \pm \\
(13 \\
\end{array}$ & $\begin{array}{r}2.42 \pm 0 \\
(30 \\
\end{array}$ & $\begin{array}{r}40 \pm 0 \\
(11 \\
\end{array}$ & $\begin{array}{r}1.12 \pm \\
(5\end{array}$ \\
\hline 750 & $\begin{array}{c}11.6 \pm 0.13 \\
(20.8)\end{array}$ & $\begin{array}{c}29.0 \pm 1.7 \mathrm{a} \\
(118.04)\end{array}$ & $\begin{array}{c}16.0 \pm 1.0 \mathrm{a} \\
(8.84)\end{array}$ & $\begin{array}{c}28.7 \pm 2.51 \mathrm{ab} \\
(82.8)\end{array}$ & $\begin{array}{c}18.1 \pm 1.25 \mathrm{a} \\
(14.55)\end{array}$ & $\begin{array}{c}2.77 \pm 0.195 \mathrm{a} \\
(48.9)\end{array}$ & $\begin{array}{c}0.41 \pm 0.058 \mathrm{a} \\
(13.88)\end{array}$ & $\begin{array}{c}1.05 \pm 0.050 \\
(-0.9)\end{array}$ \\
\hline
\end{tabular}

Note: see Table 3

Table 9. The effect of thiamin foliar application on chlorophylls, carotenoids, phenolics contents and activity of antioxidants in fenugreek leaves (in brackets percent of increase compared with control)

\begin{tabular}{cccccc}
\hline $\begin{array}{c}\text { Thiamin } \\
\begin{array}{c}\text { concentration } \\
(\mathrm{ppm})\end{array}\end{array}$ & $\begin{array}{c}\text { Chlorophyll a } \\
\left(\mathrm{mg} \cdot \mathrm{g}^{-1} \mathrm{f} . \mathrm{w} .\right)\end{array}$ & $\begin{array}{c}\text { Chlorophyll b } \\
\left(\mathrm{mg} \cdot \mathrm{g}^{-1} \mathrm{f} . \mathrm{w} .\right)\end{array}$ & $\begin{array}{c}\text { Carotenoid } \\
\left(\mathrm{mg} \cdot \mathrm{g}^{-1} \mathrm{f} . \mathrm{w} .\right)\end{array}$ & $\begin{array}{c}\text { Total phenol } \\
\left(\mathrm{mg} \cdot 100 \mathrm{~g}^{-1} \mathrm{f} . \mathrm{w} .\right)\end{array}$ & $\begin{array}{c}\text { Total anti-oxidant } \\
(\% h i b i t i o n ~ o f ~ \mathrm{DPPH})\end{array}$ \\
\hline 0 & $1.15 \pm 0.08 \mathrm{a}$ & $0.57 \pm 0.026 \mathrm{~b}$ & $0.49 \pm 0.037 \mathrm{~b}$ & $45.3 \pm 2.51 \mathrm{~b}$ & $45.7 \pm 7.5 \mathrm{~b}$ \\
\hline 250 & $\begin{array}{c}1.24 \pm 0.04 \mathrm{a} \\
(7.8)\end{array}$ & $\begin{array}{c}0.62 \pm 0.023 \mathrm{ab} \\
(8.77)\end{array}$ & $\begin{array}{c}0.54 \pm 0.028 \mathrm{ab} \\
(10.2)\end{array}$ & $\begin{array}{c}52.7 \pm 1.15 \mathrm{ab} \\
(16.3)\end{array}$ & $\begin{array}{c}53.0 \pm 3.5 \mathrm{ab} \\
(15.97)\end{array}$ \\
\hline 500 & $1.31 \pm 0.10 \mathrm{a}$ & $\begin{array}{c}0.71 \pm 0.041 \mathrm{a} \\
(24.5)\end{array}$ & $\begin{array}{c}0.58 \pm 0.020 \mathrm{a} \\
(18.36)\end{array}$ & $\begin{array}{c}56.3 \pm 4.16 \mathrm{a} \\
(24.28)\end{array}$ & $\begin{array}{c}57.3 \pm 2.0 \mathrm{ab} \\
(25.38)\end{array}$ \\
\hline 750 & $1.19 \pm 0.07 \mathrm{a}$ & $\begin{array}{c}0.63 \pm 0.047 \mathrm{ab} \\
(10.5)\end{array}$ & $\begin{array}{c}0.58 \pm 0.026 \mathrm{a} \\
(18.36)\end{array}$ & $\begin{array}{c}58.0 \pm 2.00 \mathrm{a} \\
(28.03)\end{array}$ & $\begin{array}{c}59.7 \pm 2.5 \mathrm{a} \\
(30.6)\end{array}$ \\
\hline
\end{tabular}

Note: see Table 3

\section{DISCUSSION}

Thiamine (Th) foliar application improved the vegetative and reproductive growth of coriander and fenugreek. These results are in agreement with those obtained previously by Abd El-Aziz et al. (2007) on Syngonium podophyllum (Bahuguna et al. 2012), on Oryza sativa (Farouk et al. 2012), on Lycopersicon esculentum and by Al-Abbasi et al. (2015) on Zinnia elegans. Increase in leaf, root and stem weights is due to the fact that Th is necessary for dividing meristematic stem cells and organ initial cells (Martinis et al. 2016). Moreover, the accumulation of some osmo-regulators, such as soluble sugars and free amino acids may increase in response to Th application. This phenomenon, by changing the water potential of plants, can increase the turgor pressure, which is needed for cell expansion and thereby plant growth (Sayed \& Gadallah 2002).
Th plays considerable role in carbohydrate catabolism as well as NADPH and ATP synthesis (Rapala-Kozik et al. 2012). In addition, Th derivative serves as a cofactor in enzymatic reactions of many metabolic pathways such as glycolysis, Krebs cycle, the pentose phosphate pathway, Calvin cycle, amino acid metabolism, nitrogen assimilation and synthesis of branched chain amino acid, isoprenoid and nucleic acids (Goyer 2010; Bahuguna et al. 2012; Martinis et al. 2016). Therefore, it seems that better growth and yield of coriander and fenugreek under Th application is mainly related to appropriate regulation of photosynthesis and energy-providing reactions in treated plants. Also, increase in chlorophyll content in Th-treated plants can also lead to the enhancement of photosynthesis (Hamada \& Khulaef 2000). The results of our experiments supported findings of Hamada and Khulaef (2000) on Vicia faba and Farouk et al. (2012) on Lycopersicon esculentum. 
In another study, Th application improved the chlorophyll and carotenoid contents in marigold (Soltani et al. 2014). One of the Th diphosphate-dependent pathways in the plant cell is the production of 2-deoxy-D-xylulose-5- phosphate synthase. Overexpression of this enzyme correlates with the accumulation of chlorophyll and carotenoids (Rapala-Kozik 2011; Rapala-Kozik et al. 2012). Furthermore, vitamins have an antioxidant scavenging effect, which protects chloroplasts and prevents chlorophyll degradation caused by the reactive oxygen species (Mady 2009). Additionally, the application of Th as observed in the present study, activates the synthesis of carotenoids, which protect chlorophyll from oxidation, and thereby increases the chlorophyll content (Farouk et al. 2012).

In line with previous studies on syngonium (Abd El-Aziz et al. 2007), gladiolus (Hashish et al. 2015; Sajjad et al. 2015) and tomato (Farouk et al. 2012), Th application increased the nutrient contents in coriander and fenugreek. In a study on sunflower, the contents of toxic ions $\mathrm{Na}^{+}, \mathrm{Ca}_{2}{ }^{+}$and $\mathrm{Cl}^{-}$ were significantly lower, while cytoplasmic $\mathrm{K}^{+}$content, which is essential for survival in stress condition, were higher in Th-treated plants (Sayed \& Gadallah 2002). It seems that more root expansion in Th-treated plants can be a possible reason for better absorption of nutrients in coriander and fenugreek. It has been reported that the main function of antioxidants, such as some vitamins, is the protection of cell membranes and their binding transporter proteins $\left(\mathrm{H}^{+}\right.$-ATP-ase pumps) against the reactive oxygen species and more absorption and translocation of minerals (Mady 2009). Soluble sugar accumulation, which can protect the integrity of the cell membranes might be a reason for membrane protection in the Th-treated plants (Sayed \& Gadallah 2002). Some researchers suggest that it is possible that vitamins foliar spray can stimulate the root secretion of organic acids into the soil that increase the nutrients' solubility in rhizosphere zone, which leads to uptake of more nutrients by the plant (Abd El-Aziz et al. 2007).

The effect of Th foliar application increased the total phenolic content and antioxidant activity in both coriander and fenugreek. In a similar study, the total phenolics increased in soybean plants that were treated with Th and riboflavin (Abdel-Monaim 2011). Reda et al. (2005) demonstrated that the application of Th at $60 \mathrm{ppm}$ resulted in higher content of total phenolics in thyme. Similar results were obtained on gladiolus, where phenolics increased in response to Th foliar application (Sajjad et al. 2015). Results of El-Bassiouny and Sadak (2015) in Linum usitatissimum also revealed that vitamins, ascorbic acid and $\alpha$-tocopherol induced considerable reduction in lipid peroxidation but an increase in superoxide dismutase as an antioxidant. It has been reported that Th stimulates the antioxidant defense and therefore has an important role in the protection of cells against oxidative stress (Tunc-Ozdemir et al. 2009). Moreover, Th itself can also act as a main source of antioxidants scavenging $\mathrm{O}_{2}^{-}$(or $\left.\mathrm{OH}\right)$ and thus affect the cellular response to oxidative stress (Rapala-Kozik et al. 2012; Jung \& Kim 2003).

\section{CONCLUSION}

Results of the present study revealed that foliar application of thiamin at concentrations 250, 500 and $750 \mathrm{ppm}$ were beneficial for the growth and development of coriander and fenugreek by increasing chlorophyll, phenolics, carotenoid and antioxidants compounds.

\section{Acknowledgment}

We are thankful from University of Birjand and University of Guilan for financial support for the project.

\section{REFERENCES}

Abd El-Aziz N.G., El-Quesni F.E.M., Farahat M.M. 2007. Response of vegetative growth and some chemical constituents of Syngonium podophyllum L. to foliar application of thiamine, ascorbic acid and kinetin at Nubaria. World Journal of Agricultural Sciences 3(3): 301-305.

Abdel-Monaim M.F. 2011. Role of riboflavin and thiamine in induced resistance against charcoal rot disease of soybean. African Journal of Biotechnology 10(53): 10842-10855. DOI: 10.5897/ajb11.253.

Al-Abbasi A.M.A.S., Abbas J.A., Al-Zurfi M.T.H. 2015. Effect of spraying thiamin and salicylic acid on growth and flowering of Zinnia elegans L. Advances in Agriculture and Botanics 7(1): 44-50. 
Amin M.A., Ismail M.A. 2015. Effect of indole butyric, arginine, cyanocobalamine (B12), ascorbic acid and their interactions on growth, yield and some metabolic constituents of sunflower plants. International Journal of Advanced Research in Biological Sciences 2(12): 154-162. SOI: 1.15/ijarbs-2-12-17.

Bahuguna R.N., Joshi R., Shukla A., Pandey M., Kumar J. 2012. Thiamine primed defense provides reliable alternative to systemic fungicide carbendazim against sheath blight disease in rice (Oryza sativa L.). Plant Physiology and Biochemistry 57: 159167. DOI: 10.1016/j.plaphy.2012.05.003.

Bakhshi D., Arakawa O. 2006. Induction of phenolic compounds biosynthesis with light irradiation in the flesh of red and yellow apples. Journal of Applied Horticulture 8(2): 101-104.

Boubakri H., Wahab M.A., Chong J., Bertsch C., Mliki A., Soustre-Gacougnolle I. 2012. Thiamine induced resistance to Plasmopara viticola in grapevine and elicited host-defense responses, including HR likecell death. Plant Physiology and Biochemistry 57: 120-133. DOI: 10.1016/j.plaphy.2012.05.016.

El-Awadi M.E., Abd Elbaky Y.R., Dawood M.G., Shalaby M.A., Bakry B.A. 2016. Enhancement quality and quantity of lupine plant via foliar application of some vitamins under sandy soil conditions. Research Journal of Pharmaceutical, Biological and Chemical Sciences 7(4): 1012-1024.

El-Bassiouny H.M.S., Sadak M.S. 2015. Impact of foliar application of ascorbic acid and $\alpha$-tocopherol on antioxidant activity and some biochemical aspects of flax cultivars under salinity stress. Acta Biológica Colombiana 20(2): 209-222. DOI: 10.15446/abc.v20n2.43868.

Fallahi H.R., Aminifard M.H., Jorkesh A. 2018. Effects of thiamine spraying on biochemical and morphological traits of basil plants under greenhouse conditions. Journal of Horticulture and Postharvest Research 1(1): 27-36. DOI: 10.22077/jhpr.2018.1114.1001.

Farouk S., Youssef S.A., Ali A.A. 2012. Exploitation of biostimulants and vitamins as an alternative strategy to control early blight of tomato plants. Asian Journal of Plant Sciences 11(1): 36-43. DOI: 10.3923/ajps.2012.36.43.

Goyer A. 2010. Thiamine in plants: Aspects of its metabolism and functions. Phytochemistry 71: 16151624. DOI: 10.1016/j.phytochem.2010.06.022.

Hamada A.M., Khulaef E.M. 2000. Simulative effects of ascorbic acid, thiamin or pyridoxine on Vicia faba growth and some related metabolic activities.
Pakistan Journal of Biological Sciences 3(8): 1330-1332. DOI: 10.3923/pjbs.2000.1330.1332.

Hashish K.I., Eid R.A., Kandil M.M., Mazher A.A.M. 2015. Study on various level of salinity on some morphological and chemical composition of gladiolus plants by foliar spray with glutathione and thiamine. International Journal of ChemTech Research 8(9): 334-341.

Hassan F.A.S., Mahfouz S.A. 2012. Effect of 1-methylcyclopropene (1-MCP) on the postharvest senescence of coriander leaves during storage and its relation to antioxidant enzyme activity. Scientia Horticulturae 141: 69-75. DOI: 10.1016/j.scienta.2012.04.021.

Jones J.B. Jr. 2001. Laboratory Guide for Conducting Soil Tests and Plant Analysis. CRC Press, Boca Raton, USA, 384 p. DOI: 10.1201/9781420025293.

Jung I.L., Kim I.G. 2003. Thiamine protects against paraquat-induced damage: scavenging activity of reactive oxygen species. Environmental Toxicology and Pharmacology 15: 19-26. DOI: 10.1016/j.etap.2003.08.001.

Kakani R.K., Anwer M.M. 2012. Fenugreek. In: Peter K.V. (Ed.), Handbook of herbs and spices, $2^{\text {nd }}$ ed., vol. 1. Woodhead Publishing, UK, pp. 286-298. DOI: $10.1533 / 9780857095671.286$.

Mady M.A. 2009. Effect of foliar application with salicylic acid and vitamin E on growth and productivity of tomato (Lycopersicon esculentum, Mill.) plant. Journal of Agricultural Sciences, Mansoura University 34(6): 6735-6746.

Mandal S., DebMandal M. 2016. Fenugreek (Trigonella foenum-graecum L.) oils. In: Preedy V.R. (Eds.), Essential Oils in Food Preservation, Flavor and Safety. Elsevier, pp. 421-429. DOI: 10.1016/b9780-12-416641-7.00047-x.

Martinis J., Gas-Pascual E., Szydlowski N., Crèvecoeur M., Gisler A., Bürkle L., Fitzpatrick T.B. 2016. Long-distance transport of thiamine (vitamin $\mathrm{B}_{1}$ ) is concomitant with that of polyamines. Plant Physiology 171: 542-553. DOI: 10.1104/pp.16.00009.

Mínguez-Mosquera M.I., Pérez-Gálvez A. 1998. Color quality in Paprika oleoresins. Journal of Agricultural and Food Chemistry 46(12): 5124-5127. DOI: 10.1021/jf980728n.

Neffati M., Marzouk B. 2008. Changes in essential oil and fatty acid composition in coriander (Coriandrum sativum L.) leaves under saline conditions. Industrial Crops and Products 28: 137-142. DOI: 10.1016/j.indcrop.2008.02.005. 
Ranjbar B., Sharafzadeh S., Alizadeh O. 2014. Growth and essential oil responses of German chamomile to thiamine and ascorbic acid. Bulletin of Environment, Pharmacology and Life Sciences 3(7): 51-53.

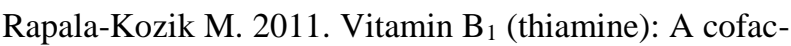
tor for enzymes involved in the main metabolic pathways and an environmental stress protectant. Advances in Botanical Research 58: 37-91. DOI: 10.1016/b978-0-12-386479-6.00004-4.

Rapala-Kozik M., Wolak N., Kujda M., Banas A.K. 2012. The upregulation of thiamine (vitamin $B_{1}$ ) biosynthesis in Arabidopsis thaliana seedlings under salt and osmotic stress conditions is mediated by abscisic acid at the early stages of this stress response. Plant Biology 12(2), 14 p. DOI: 10.1186/1471-2229-12-2.

Raschke M., Bürkle L., Müller N., Nunes-Nesi A., Fernie A.R., Arigoni D. et al. 2007. Vitamin B1 biosynthesis in plants requires the essential iron-sulfur cluster protein, THIC. Proceedings of the National Academy of Sciences of the United States of America 104: 19637-19642. DOI: 10.1073/pnas.0709597104.

Reda F., Abdel-Rahim E.A., El-Baroty G.S.A., Ayad H.S. 2005. Response of essential oils, phenolic components and polyphenol oxidase activity of thyme (Thymus vulgaris, L.) to some bioregulators and vitamins. International Journal of Agriculture and Biology 7(5): 735-739.

Sajjad Y., Jaskani M.J., Qasim M., Akhtar G., Mehmood A. 2015. Foliar application of growth bioregulators influences floral traits, corm-associated traits and chemical constituents in Gladiolus grandiflorus L. Korean Journal of Horticultural Science and Technology 33(6): 812-819. DOI: 10.7235/hort.2015.15052.

Sánchez-Moreno C., Larrauri J.A., Saura-Calixto F. 1999. A procedure to measure the antiradical efficiency of polyphenols. Journal of the Science of Food and Agriculture 76(2): 270-276. DOI: 10.1002/(sici)1097-0010(199802)76:2<270::aidjsfa945>3.0.co;2-9.

Sayed S.A., Gadallah M.A.A. 2002. Effects of shoot and root application of thiamin on salt-stressed sunflower plants. Plant Growth Regulation 36(1): 7180. DOI: 10.1023/a:1014784831387.

Soltani Y., Saffari V.R., Maghsoudi Moud A.A. 2014. Response of growth, flowering and some biochemical constituents of Calendula officinalis L. to foliar application of salicylic acid, ascorbic acid and thiamine. Ethno-Pharmaceutical Products 1(1): 37-44.

Tavarini S., Degl'Innocenti E., Remorini D., Massai R., Guidi L. 2008. Antioxidant capacity, ascorbic acid, total phenols and carotenoids changes during harvest and after storage of Hayward kiwifruit. Food Chemistry 107(1): 282-288. DOI: 10.1016/j.foodchem.2007.08.015.

Tunc-Ozdemir M., Miller G., Song L., Kim J., Sodek A., Koussevitzky S. et al. 2009. Thiamin confers enhanced tolerance to oxidative stress in Arabidopsis. Plant Physiology 151: 421-432. DOI: 10.1104/pp.109.140046. 